\title{
PARALLEL FRAMINGS AND FOLIATIONS ON PSEUDORIEMANNIAN MANIFOLDS
}

\author{
S. A. ROBERTSON \& P. M. D. FURNESS
}

This paper is in two main parts. The first part is concerned with the global geometrical and topological structure of a pseudoriemannian $m$-manifold which is foliated by the integral manifolds of a parallel field of tangent $k$-planes. The second part deals with the more restrictive situation in which $k$ mutually orthogonal parallel vector fields are defined on the manifold.

The main results depend on the local canonical forms for the metric tensor given by Walker [15], [16]. The basic method is to examine the form of the transformations of local coordinates for the canonical charts of Walker, from which it is possible to obtain strong restrictions on the structure of the leaves of the foliations, the vector fields, and the parent manifold. For other applications of this method, see Robertson [10] and Furness and Willmore [1].

Thanks are due to I. R. Porteous for observations embodied in $\S 1$, which have greatly simplified the proof of Lemma 2 . Also, the referee has drawn our attention to an interesting analogy with recent work of Weinstein [20] on Lagrangian foliations.

\section{Pseudoriemannian metrics}

Let $E$ be a real $m$-plane bundle over a topological space $X$, with projection $p: E \rightarrow X$. Recall that a Riemannian metric $\rho$ in $E$ assigns to each $x \in X$ a symmetric positive-definite bilinear form $\rho_{x}: E_{x} \times E_{x} \rightarrow R$, where $E_{x}=p^{-1}(x)$ is the fibre over $x$, and the forms $\rho_{x}$ vary continuously with $x$.

More generally, if we relax the requirement that $\rho_{x}$ be positive-definite to the condition that $\rho_{x}$ be nondegenerate, then $\rho$ is called a pseudoriemannian metric in $E$. The signature of $\rho_{x}$ is then independent of $x$ if $X$ is connected, and is called the signature of $\rho$ in this case. For convenience, we use the term signature to mean the ordered pair $(j, m-j)$, where $\rho_{x}$ has $j$ negative and $m-j$ positive eigenvalues.

Suppose now that $F$ is a subbundle of $E$ of fibre dimension $r$ such that $\rho_{x} \mid F_{x}=0$, that is, $\rho_{x}(\lambda, \mu)=0$ for all $\lambda, \mu \in F_{x}$. The orthogonal complement $G$ of $F$ in $E$ is then a subbundle of $E$ of fibre dimension $m-r$ such that $F_{x} \subset G_{x}$ for all $x \in X$, with

Communicated by W. P. A. Klingenberg, January 2, 1973. 


$$
G_{x}=\left\{\xi \in E_{x}: \forall \eta \in F_{x}, \rho_{x}(\xi, \eta)=0\right\}
$$

Now consider the quotient bundle $H=E / G$. Both $H$ and its dual $H^{*}$ are $r$ plane bundles over $X$, with $H_{x}^{*}=L\left(H_{x}, R\right)$.

Lemma 1. There is a bundle-isomorphism

$$
\theta: F \rightarrow H^{*}
$$

Proof. For each $x \in X$, define $\theta_{x}: F_{x} \rightarrow H_{x}^{*}$ by $\theta_{x}(\lambda)(\psi)=\rho_{x}(\lambda, \mu)$, where $\mu \in E_{x}$ is any representative of $\psi \in H_{x}=E_{x} / G_{x}$. It is trivial to check that $\theta_{x}$ is an isomorphism and determines fibre-wise the required bundle-isomorphism $\theta$.

Corollary 1. For some $(m-2 r)$-plane bundle $K$ on $X$ there is a bundleisomorphism

$$
E \cong F \oplus F \oplus K
$$

Proof. By the Lemma, $E \cong F \oplus H^{*} \oplus K \simeq F \oplus F \oplus K$.

Corollary 2. If $m=2 r$, then $E$ admits an almost-complex structure. Moreover, the Stiefel-Whitney classes of $E$ are given by

$$
W_{2 i+1}(E)=0, \quad W_{2 i}(E)=W_{i}(F)^{2} .
$$

Proof. By Corollary $1, E \cong F \oplus F$. Define $J_{x}: E_{x} \rightarrow E_{x}$, where $E_{x}=$ $F_{x} \oplus F_{x}$ by $J(\lambda, \mu)=(-\mu, \lambda)$. Then $J$ is an almost-complex structure on $E$. The remaining statements follow immediately from the relation $W(E)=$ $W(F \oplus F)=W(F) W(F)$.

\section{Parallel fields of planes}

Suppose that $M$ is a smooth $\left(=C^{\infty}\right)$ connected complete $m$-dimensional pseudoriemannian manifold, that is to say, $M$ is a Riemann m-manifold. In the theory of $\S 1$ we take $X=M$ and $E=T M$, and suppose that $\rho_{x}$ varies smoothly with $x \in M$. Let $P$ be a smooth subbundle of $T M$, of fibre-dimension $k$. We say that $P$ is parallel if for each $x, y \in M$, each $\lambda \in P_{x}$ and each smooth path $\gamma: I \rightarrow M$ from $x$ to $y$, the element of $T_{y} M$ obtained by parallel transport of $\lambda$ along $\gamma$ lies on $P_{y}$.

The following facts are standard (see, for example, Walker [15]):

(i) The orthogonal complement $Q$ of $P$ in $T M$ is a parallel subbundle of dimension $m-k$.

(ii) Further parallel subbundles $P+Q$ and $P \cap Q$ of dimensions $m-r$ and $r$ respectively are given by

$$
(P+Q)_{x}=P_{x}+Q_{x}, \quad(P \cap Q)_{x}=P_{x} \cap Q_{x} .
$$


Moreover, $P \cap Q$ is null in the sense that $\rho_{x} \mid P_{x} \cap Q_{x}=0$ for all $x \in M$.

The results of $\S 1$ now apply with $F=P \cap Q$ and $G=P+Q$, since $P+Q$ is the orthogonal complement of $P \cap Q$. Thus we have, as a special case of Lemma 1,

Lemma 2. There is a bundle-isomorphism

$$
\theta: P \cap Q \rightarrow(T M /(P+Q))^{*} .
$$

Any parallel subbundle $P$ of $T M$, considered as a parallel field of tangent $k$-planes, is integrable and is therefore the tangent field of a foliation $\mathscr{F}$, which we call a parallel foliation of $M$. The leaves of $\mathscr{F}$ are totally geodesic (complete) submanifolds of $X$. Any such parallel foliation $\mathscr{F}$ determines three other foliations $\mathscr{F}_{\perp}, \mathscr{F}_{n}$ and $\mathscr{F}_{+}$whose tangent fields are $Q, P \cap Q$ and $P+Q$ respectively. It is convenient to put $k=r+s$ and to say that $P$ or $F$ is of type $(r, s)$. We say that $P$ and $\mathscr{F}$ are nondegenerate if $r=0$, degenerate if $r>0$, partially degenerate if $r>0$ and $s>0$, and totally degenerate (or null) if $s=0$. It should be noted that $\mathscr{F}_{\perp}$ and $Q$ are (non-) degenerate if and only if $\mathscr{F}$ is (non-) degenerate.

\section{Walker atlases}

Let $\mathscr{F}$ be a parallel foliation of type $(r, s)$ on the Riemann $m$-manifold $M$. We identify $R^{m}$ with the product $R^{r} \times R^{s} \times R^{u} \times R^{r}$, where $u=m-2 r-s$, and use variables $x, y, z, t$ in this space. Thus $R^{m}$ has foliations $\mathscr{F}^{\prime}, \mathscr{F}_{\perp}^{\prime}, \mathscr{F}_{n}^{\prime}$, $\mathscr{F}_{+}^{\prime}$ whose leaves are planes of the form $R^{r} \times R^{s} \times\{z\} \times\{t\}, R^{r} \times\{y\} \times R^{u}$ $\times\{t\}, R^{r} \times\{y\} \times\{z\} \times\{t\}$ and $R^{r} \times R^{s} \times R^{u} \times\{t\}$ respectively.

An admissible chart $\xi: U \rightarrow U^{\prime}$ on $M$ is said to be $\mathscr{F}$-related if it is an isomorphism of foliations from $P \mid U$ onto $P^{\prime} \mid U^{\prime}$, where $P=\mathscr{F}, \mathscr{F}_{\perp}, \mathscr{F}_{n}$ and $\mathscr{F}_{+}$. It was shown by Walker [16] that $M$ admits an atlas of $\mathscr{F}$-related charts $\xi: U \rightarrow U^{\prime}$ in which, for each $p \in U$, the matrix $M(p)$ of $\rho_{p}$ in $\xi$-coordinates has the form

$$
M(p)=\left[\begin{array}{cccc}
0 & 0 & 0 & I \\
0 & A(y, t) & 0 & H(y, t) \\
0 & 0 & B(z, t) & K(z, t) \\
I & H^{\dagger}(y, t) & K^{\dagger}(z, t) & C(x, y, z, t)
\end{array}\right]
$$

where: $I$ is the unit $r \times r$ matrix; $A, B$ and $C$ are symmetric; $\dagger$ denotes transposition; both $A$ and $B$ are invertible; and the partition of $M(p)$ conforms to the above product decomposition of $R^{m}$. Walker also observed that if $M$ admits such an atlas $\mathscr{A}$, then it has a parallel foliation $\mathscr{F}$ of type $(r, s)$ for which each chart is $\mathscr{F}$-related. For simplicity, we call such an atlas $\mathscr{A}$ a Walker atlas for $\mathscr{F}$ (the Riemann tensor field $\rho$ being fixed).

Suppose then that $\mathscr{A}$ is a Walker atlas for $\mathscr{F}$, and let $\xi: U \rightarrow U^{\prime}$ and 
$\eta: V \rightarrow V^{\prime}$ be $\mathscr{A}$-charts with $W=U \cap V \neq \emptyset$. A straightforward calculation shows that the transformation of coordinates $\theta_{\xi \eta}: \xi(W) \rightarrow \eta(W)$,

$$
\xi(w)=(x, y, z, t) \rightarrow\left(x_{*}, y_{*}, z_{*}, t_{*}\right)=\eta(w)
$$

for all $w \in W$, is given by equations of the form

$$
\begin{aligned}
& x_{*}=\left(d T(t)^{-1}\right)^{\dagger}(x)+f(y, t)+g(z, t), \\
& y_{*}=Y(y, t), \quad z_{*}=Z(z, t), \quad t_{*}=T(t),
\end{aligned}
$$

where the differentials $d T(t), d_{1} Y(y, t)$ and $d_{1} Z(z, t)$ are invertible linear automorphisms of $R^{r}, R^{s}$ and $R^{u}$ respectively, and $\dagger$ denotes the adjoint with respect to the standard inner product on $R^{r}$.

Our aim is to deduce information on the global structure of $M$ from these equations. Since each leaf of $\mathscr{F}$ is given locally in terms of $\xi$-coordinates by $t=$ constant, $y=$ constant, $z=$ constant, we obtain at once the following important fact.

Theorem 1. Let $\mathscr{F}$ be a parallel foliation of $M$. Then $M$ admits an atlas whose restriction to each leaf of $\mathscr{F}_{n}$ is a flat affine atlas on that leaf.

Thus the leaves of $\mathscr{F}_{n}$ admit flat affine structures and in particular have $R^{r}$ as universal covering space.

As this stage, we also note that Lemma 2 can be interpreted in terms of foliations, as follows. If $\mathscr{P}$ is a foliation of $M$, then the bundle of planes tangent to the leaves of $\mathscr{P}$ is denoted by $\tau \mathscr{P}$, and the quotient bundle $\tau M / \tau \mathscr{P}$ by $\nu \mathscr{P}$. We call $\tau \mathscr{P}$ and $\nu \mathscr{P}$ the tangent bundle and normal bundle of $\mathscr{P}$. With these conventions, Lemma 2 and the corollary of Lemma 1 yield

Theorem 2. Let $\mathscr{F}$ be a parallel foliation on $M$. Then there is a vector bundle isomorphism

$$
\theta: \nu * \mathscr{F}_{+} \rightarrow \tau \mathscr{F}_{\cap} .
$$

Corollary. Let $\mathscr{F}$ be a parallel foliation of type $(r, 0)$ on a $2 r$-manifold $M$. Then $M$ admits an almost-complex structure, there is a vector bundle isomorphism $\tau M \cong \tau \mathscr{F} \oplus \tau \mathscr{F}$, and the Stiefel-Whitney classes of $M$ are given by

$$
W_{2 i+1}(M)=0, \quad W_{2 i}(M)=W_{i}(\tau)^{2} .
$$

Remarks. (1) Let $M=X \times Y$, where $X$ and $Y$ are parallelisable $m$ manifolds, and let $\mathscr{F}$ be the foliation of $M$ by the factors $X \times\{y\}$. Then $\tau M \cong \tau \mathscr{F} \oplus \tau \mathscr{F}$. However, $M$ need not have any Riemann structure for which $\mathscr{F}$ is null and parallel. For instance, if $X=Y=S^{3}$, then Theorem 1 implies that no such Riemann structure exists.

(2) The Stiefel-Whitney classes of $M=P_{2 n+1}(C)$ are "almost" of the above form in that they vanish in odd dimensions and are squares in even dimensions. But $\tau P_{2 n+1}(C)$ is not expressible as a square. 
(3) Let $M$ be the cotangent space $T^{*} X$ of an $m$-manifold $X$, and $\mathscr{F}$ the foliation of $M$ by the fibres $T_{p}^{*} X$. Then $M$ admits a Riemann structure for which $\mathscr{F}$ is null and parallel (see Patterson and Walker [5]).

\section{Fibrings}

To progress further we suppose that $\mathscr{F}$ is a parallel foliation of type $(r, s)$ on $M$ for which $\mathscr{F}_{n}$ is a fibring of $M$. Thus $M_{n}=M / \mathscr{F}_{n}$ is an $(m-r)$ manifold, and the quotient map $f_{\cap}: M \rightarrow M_{\cap}$ is such that $(P \cap Q)_{p}=\operatorname{ker} T f_{\cap}(p)$ for all $p \in M$.

The map $f_{n}$ induces foliations $\mathscr{G}, \mathscr{G}_{\perp}$ and $\mathscr{G}_{+}$on $M_{\mathrm{n}}$, defined by $\mathscr{F}\left(f_{n}(x)\right)$ $=f_{\cap}(\mathscr{F}(x))$, etc. The dimensions of $\mathscr{G}, \mathscr{G}_{\perp}$ and $\mathscr{G}_{+}$are therefore $s, u$ and $m-2 r$, where $u=m-2 r-s$ as before. We note that $\psi+\psi_{\perp}=\psi_{+}$, where $\psi_{,} \psi_{\perp}$ and $\psi_{+}$are the tangent bundles of $G, G_{\perp}$ and $G_{+}$respectively.

Our aim is to show that the fibres of $\mathscr{F}_{\cap}$ are Euclidean cylinders [2, p. 210], and that the leaves of $\mathscr{G}_{+}$are covered by Riemann products.

First we observe that there is a $\mathscr{G}$-related atlas $\mathscr{B}$ on $M$ such that for each $\mathscr{B}$-chart $\eta: V \rightarrow V^{\prime}$ there is a fibre-preserving diffeomorphism from $V \times F_{\mathrm{n}} \rightarrow$ $f_{n}^{-1}(V)$, where $F_{n}$ is the fibre of $\mathscr{F}_{n}$. It now follows immediately from Walker's construction [16] that there is a Walker atlas $\mathscr{A}$ for $\mathscr{F}$ having the following properties. If $\xi: U \rightarrow U^{\prime}$ is an $\mathscr{A}$-chart, then there is a $\mathscr{B}$-chart $\eta: V \rightarrow V^{\prime}$ such that $V=f_{\cap}(U)$ and $\pi(\xi(p))=\eta\left(f_{\cap}(p)\right)$ for all $p \in U$, where $\pi: R^{m}=R^{r}$ $\times R^{m-r} \rightarrow R^{m-r}$ is projection from the first factor. We say that $\eta$ is a projection of $\xi$, and $\xi$ is a lift on $\eta$ in this case. Secondly, the set of all $\mathscr{B}$-charts $\xi_{i}: U_{i} \rightarrow U_{i}^{\prime}$ which are lifts of any $\mathscr{B}$-chart $\eta: V \rightarrow V^{\prime}$ is such that $\bigcup_{i} U_{i}=$ $f_{n}^{-1}(V)$. We denote this set of $\mathscr{A}$-charts by $\mathscr{A}(\eta)$. The essential point to note is that the coordinates $x$ are defined in terms of $y, z$ and $t$. The complete argument can be found in [16]. We say that the Walker atlas $\mathscr{A}$ covers $\mathscr{B}$.

Theorem 3. Let $\mathscr{F}$ be a parallel foliation of $M$ such that $\mathscr{F}_{n}$ is a fibring. Then there is an atlas $\mathscr{A}$ on $M$ whose restriction to each leaf $\mathscr{F}_{n}(p)$ of $\mathscr{F}_{n}$ is a flat affine atlas with respect to which $\mathscr{F}_{n}(p)$ is affinely equivalent to a Euclidean cylinder.

Proof. Let $\mathscr{A}$ be a Walker atlas for $\mathscr{F}$ covering an atlas $\mathscr{B}$ of $M_{n}$. Let $p \in M$ and $q=f(p)$. Then there is a $\mathscr{B}$-chart $\eta: V \rightarrow V^{\prime}$ with $q \in V^{\prime}$. Let $\xi_{i}: U_{i} \rightarrow U_{i}^{\prime}, \xi_{j}: U_{j} \rightarrow U_{j}^{\prime}$ be elements of $\mathscr{A}(\eta)$, with $W=U_{i} \cap U_{j} \neq \emptyset$. Then the equations relating $\xi_{i}$-coordinates $(x, y, z, t)$ to $\xi_{j}$-coordinates $\left(x_{*}, y_{*}, z_{*}, t_{*}\right)$ of $w \in W$ take the simplified form

$$
x_{*}=x+h_{i j}, \quad\left(y_{*}, z_{*}, t_{*}\right)=(y, z, t)=\eta(q),
$$

for some $h_{i j} \in R^{r}$. Hence $\mathscr{F}_{n}(p)$ has an atlas $\mathscr{A}(\eta) \mid \mathscr{F}_{n}(p)$ in which all coordinate transformations are translations, which proves the theorem.

Theorem 4. Let $F$ be a parallel foliation of $M$ such that $\mathscr{F}_{\cap}$ is a fibring. 
Then each leaf $\mathscr{G}_{+}(v)$ of the induced foliation $\mathscr{G}_{+}$of $M_{n}$ has an induced Riemann metric for which $\mathscr{G}$ and $\mathscr{G}_{\perp}$ are conjugate nondegenerate parallel foliations.

Proof. Again let $\mathscr{A}$ be a Walker atlas for $\mathscr{F}$ covering an atlas $\mathscr{B}$ of $\boldsymbol{M}_{\mathrm{n}}$, and for $q \in M_{n}$ let $\mathscr{B}(q)$ denote the restriction of $\mathscr{B}$ to $\mathscr{G}_{+}(q)$. Then, with $\mathscr{H}$ $=\mathscr{G} \mid \mathscr{G}_{+}(q)$, the $\mathscr{B}(q)$-charts are $\mathscr{H}$-related, and the matrix of the induced symmetric bilinear form is

$$
N(q)=\left[\begin{array}{cc}
A(y, t) & 0 \\
0 & B(z, t)
\end{array}\right]
$$

on $\mathscr{B}(q)$-charts, which proves the theorem.

Corollary. With the hypotheses of Theorem 4 , the universal covering $\tilde{\mathscr{G}}_{+}(q)$ of $\mathscr{G}_{+}(q)$ is the Riemann product of the s-manifold $\tilde{\mathscr{G}}(q)$ with the Riemann $u$-manifold $\tilde{\mathscr{G}}_{\perp}(q)$.

Proof. This follows at once from Wu's theorem [18].

\section{Parallel foliations of type $(n, 0)$}

In this section we consider a parallel foliation $\mathscr{F}$ of dimension $n$ on a Riemann $2 n$-manifold $M$, of type $(n, 0)$. Thus $\mathscr{F}$ is totally degenerate (i.e., null), so that $\mathscr{F}=\mathscr{F}_{\perp}=\mathscr{F}_{n}=\mathscr{F}_{+}$. To suppose that $\mathscr{F}$ fibres $M$, therefore, allows us to use the results of $\S 4$ with $\mathscr{F}=\mathscr{F}_{n}$.

Of course the induced foliations $\mathscr{G}, \mathscr{G}_{\perp}$ and $\mathscr{G}_{+}$of the base $Y=M_{n}$ degenerate here to the trivial foliation of $Y$ by its points, and Theorem 4 yields no information in this case. By Theorem 3, however, $\mathscr{F}$ has a Euclidean cylinder $F=\left(S^{1}\right)^{a} \times R^{b}$ as fibre, $a+b=n$, and each fibre $\mathscr{F}(p)=f^{-1}(q)$ is affinely equivalent to $F$, where $f: M \rightarrow Y$ is the projection, $f(p)=q$, and $\mathscr{F}(p)$ has the flat affine structure determined by any Walker atlas for $\mathscr{F}$.

In particular, if $F=R^{n}$, then $\mathscr{F}$ is an affine bundle over $Y$, and hence $M$ is the total space of a vector bundle over $Y$. If follows immediately from our equations relating local coordinates that this vector bundle is equivalent to $\tau^{*} Y$. More precisely, we obtain

Theorem 5. Let $\mathscr{F}$ be a parallel null fibring of a Riemann $2 n$-manifold $M$ with simply connected fibre and n-dimensional base $Y$. Then there is an affine bundle isomorphism from $\mathscr{F}$ to the cotangent bundle of $Y$.

\section{Parallel framings}

An orthogonal $k$-frame at $x \in M$ is an ordered set $\lambda=\left(\lambda_{1}, \cdots, \lambda_{k}\right)$ of mutually orthogonal, linearly independent tangent vectors to $M$ at $x$. The set of all orthogonal $k$-frames at $x \in M$ forms a Stiefel manifold $S_{x}^{k}$ which is the fibre over $x$ of the Stiefel bundle $S^{k} M$. A smooth section $\sigma$ of $S^{k} M$ is called a $k$ framing of $M$, and determines an ordered set $\left(\sigma_{1}, \cdots, \sigma_{k}\right)$ of smooth, linearly 
independent, mutually orthogonal vector fields $\sigma_{i}$. The section $\sigma$ also determines a subbundle $\Sigma$ of $\tau M$ generated by $\sigma_{1}, \cdots, \sigma_{k}$.

The framing $\sigma$ is said be parallel of type $(r, k-r)$ if:

(1) $\forall i=1, \cdots, k, \sigma_{i}$ is a parallel vector field;

(2) $\sigma_{1}, \cdots, \sigma_{r}$ are null;

(3) $\sigma_{r+1}, \cdots, \sigma_{k}$ are nonnull and unit;

(4) $\sigma_{1}, \cdots, \sigma_{r}$ generate $\Sigma \cap \Sigma_{\perp}$.

We note that if $\sigma$ is parallel, then $\Sigma$ is a strictly parallel field of $k$-planes of nullity $r$, in the terminology of [15]. The results of previous sections can now be strengthened considerably for such parallel fields. As before we denote the foliations determined by $\Sigma, \Sigma_{\perp}, \Sigma \cap \Sigma_{\perp}$ and $\Sigma+\Sigma_{\perp}$ by $\mathscr{F}, \mathscr{F}_{\perp}, \mathscr{F}_{+}, \mathscr{F}_{n}$ respectively.

Suppose that $\sigma$ is a parallel $k$-framing of $M$ of type $(r, k-r)$. Then in the notation of $\S 3$ there is a Walker atlas $\mathscr{A}$ on $M$ such that the matrix of the metric tensor has the form

$$
\left[\begin{array}{cccc}
0 & 0 & 0 & I_{r} \\
0 & A & 0 & 0 \\
0 & 0 & B(z, t) & G(z, t) \\
I_{r} & 0 & G^{\dagger}(z, t) & C(z, t)
\end{array}\right]
$$

where $A, B, C$ are symmetric matrices of order $s \times s, u \times u$, and $r \times r$ respectively, where $r+s=k$ and $u+r=m-k$. Also, $A$ and $B$ are invertible, and $A$ is a constant diagonal matrix with entries of the form \pm 1 . Each chart has coordinates $(x, y, z, t) \in R^{r} \times R^{s} \times R^{u} \times R^{r}$ and with respect to these coordinates we have:

$$
\sigma_{1}=\frac{\partial}{\partial x^{1}}, \cdots, \sigma_{r}=\frac{\partial}{\partial x^{r}}, \sigma_{r+1}=\frac{\partial}{\partial y^{1}}, \cdots, \sigma_{k}=\frac{\partial}{\partial y^{s}} .
$$

It follows that the variables $\left(x_{*}, y_{*}, z_{*}, t_{*}\right),(x, y, z, t)$ in the overlap of two charts are related by equations of the form

$$
x_{*}=x+\alpha(z, t), \quad y_{*}=y+\beta, \quad z_{*}=Z(z, t), \quad t_{*}=t+\gamma,
$$

where $\beta \in R^{s}, \gamma \in R^{r}$ are constants, and $Z, \alpha$ are smooth functions of the coordinates $z, t$.

The existence of $\mathscr{A}$ leads to the following result.

Theorem 6. Let $M$ be a Riemann m-manifold with a parallel $k$-framing $\sigma$ of type $(r, k-r)$.

(i) Then $\tau M \simeq \iota^{k+r} \oplus \xi$ for some subbundle $\xi$ of $\tau(M)$.

(ii) If $M$ is closed, then it is a bundle over the torus $T^{k}$, and the leaves of $\mathscr{F}$ and $\mathscr{F}_{n}$ are affinely equivalent in the induced structure to euclidean cylinders. Furthermore there is a $k$-dimensional subspace in $H^{1}(M ; R)$. 
Proof. (i) follows immediately from Theorem 2.

(ii) $d t=\left(d t^{1}, \cdots, d t^{r}\right)$ determines $r$ independent closed nonvanishing 1forms. It follows that $M$ is a bundle over $T^{k}$ by Theorem 1 of Tischler [14]. Also $d t$ determines an $r$-dimensional linear subspace in $H^{1}(M ; R)$. It should be noted that this fibring need not coincide with $\mathscr{F}$.

\section{Parallel framings of maximum nullity}

The extreme case of parallel framings of type $(r, 0)$ on manifolds of dimension $m=2 r$ or $(2 r+1)$ is now considered. The metric of $M$ has signature $(r, r)$ if $m$ is even, and $(r+1, r)$ or $(r, r+1)$ if $m$ is odd. If $m$ is even, it follows immediately from Theorem 6 that $M$ is parallelizable.

Theorem 7. Let $M$ be a smooth connected complete Riemann $2 r$ - or $(2 r+1)$-manifold with a parallel framing of type $(r, 0)$. Then, for all $x \in M$, $\exp _{x}: M_{x} \rightarrow M$ is a covering map.

Proof. Case 1: $M$ is even dimensional. There is a Walker atlas $\mathscr{A}$ on $M$ such that in each chart the metric tensor has the form

$$
\left(g_{i j}\right)=\left[\begin{array}{cc}
0 & I_{r} \\
I_{r} & C(t)
\end{array}\right] .
$$

Each chart has coordinates $(x, t) \in R^{r} \times R^{r}$, and on the overlap of two charts the coordinates $\left(x_{*}, t_{*}\right),(x, t)$ are related by equations of the form

$$
\begin{aligned}
x_{*} & =x+\alpha(t), \\
t_{*} & =t+\gamma, \quad \text { where } \gamma \in R^{r} \text { is constant. }
\end{aligned}
$$

For ease of notation $x$ will be denoted by $x^{\lambda}, \lambda=1, \cdots, r$, and $t$ by $t^{1}=x^{r+1}$, $\cdots, t^{r}=x^{2 r}$. Late Greek suffices $\lambda, \mu, \tau, \cdots$ will denote integers in $(1, \cdots, r)$, early Greek suffices $\alpha, \beta, \gamma, \cdots$ integers in $(r+1, \cdots, 2 r)$, and Roman suffices $i, j, k, \cdots$ integers in $(1, \cdots, 2 r)$. The coefficients of the Levi-Civita connexion satisfy

$$
\Gamma_{j k}^{\alpha}=\Gamma_{\mu k}^{i}=0, \quad \Gamma_{\beta \gamma}^{\lambda}=\frac{1}{2} g^{2 \alpha}\left(g_{\alpha \beta, \gamma}+g_{\alpha \gamma, \beta}-g_{\beta \gamma, \alpha}\right) .
$$

The equations for a geodesic $\theta:[0,1] \rightarrow M$ reduce to

$$
\frac{d^{2} \theta^{\lambda}}{d u^{2}} \Gamma_{\alpha \beta}^{\lambda}\left(\theta^{\alpha}(u)\right) X^{\alpha} X^{\beta}=0, \quad \frac{d^{2} \theta^{\alpha}}{d u^{2}}=0,
$$

where $\left(d \theta^{\lambda} / d u\right)(0)=X^{\lambda}$ and $\left(d \theta^{\alpha} / d u\right)(0)=X^{\alpha}$.

Let $x \in M$ and $X_{0} \in M_{x}$. Let $\theta:[0,1] \rightarrow M$ be the geodesic determined by $X_{0}$ such that $\theta(1)=\exp _{x} X_{0}$. Cover $\theta([0,1])$ with charts $U_{0}, \cdots, U_{N}$ of $\mathscr{A}$ for which there is a subdivision $\left[0, u_{1}\right], \cdots,\left[u_{i}, u_{i+1}\right], \cdots,\left[u_{N-1}, 1\right]$ of $[0,1]$ satisfying $\theta\left(\left[u_{i}, u_{i+1}\right]\right) \subset U_{i}$. 
Suppose that $X_{0}$ has components $X_{0}^{j}$ with respect to the chart $U_{0}$. It follows from (2) that in the chart $U_{i}, \theta$ has coordinates

$$
\begin{gathered}
\theta_{i}^{\alpha}(u)=\frac{d \theta_{i}^{\lambda}}{d u}\left(u_{i}\right)\left(u-u_{i}\right)+\theta_{i}^{\alpha}\left(u_{i}\right)-X_{0}^{\alpha} X_{0}^{\beta} \int_{u_{i}}^{u} \int_{u_{i}}^{v} i^{\Gamma_{\alpha \beta}^{\alpha}}\left(\theta_{i}^{\gamma}(s)\right) d s d v, \\
\theta_{i}^{\alpha}(u)=X_{0}^{\alpha}\left(u-u_{i}\right)+\theta_{i}^{\alpha}\left(u_{i}\right) .
\end{gathered}
$$

By using an inductive argument with (1), we can obtain

$$
\theta_{i}^{\lambda}(u)=X_{0}^{\lambda} u+Q_{i}^{\lambda}\left(u, X_{0}^{\alpha}\right), \quad \theta_{i}^{\alpha}(u)=X_{0}^{\alpha} u+A_{i}^{\alpha},
$$

where $A_{i}^{\alpha}$ is constant and $Q_{i}^{\alpha}$ is smooth. Thus in the chart $U_{N}$ we can represent $\exp _{x} X_{0}$ by

$$
\left(\exp _{x} X_{0}\right)^{\alpha}=\theta_{N}^{\alpha}(1), \quad\left(\exp _{x} X_{0}\right)^{\lambda}=\theta_{N}^{\alpha}(1) .
$$

It is clear from (3) that the Jacobian of this map has the matrix form

$$
\left[\begin{array}{cc}
I_{r} & P\left(X_{0}\right) \\
0 & I_{r}
\end{array}\right] \quad \text { for some smooth } P \text {. }
$$

This matrix is nonsingular, and so $\exp _{x}$ is a local diffeomorphism. It follows that there are a neighborhood $W$ of $X_{0}$ in $M_{x}$ and an open set $U \subset U_{N}$ such that $\exp _{x}: W \rightarrow U$ is a diffeomorphism. We now show that $\exp _{x}$ is onto $\bar{U}$ (the closure of $U$ in $\left.U_{N}\right)$. Let $z=\left(x^{\alpha}, x^{\alpha}\right)$ be a limit point of $U$ in $U_{N}$, and let $X(p) \in W, p=1,2,3, \cdots$, be a sequence such that $\exp _{x} X(p)$ converges to $z$. Clearly $X(p)$ converges to $x^{\alpha}-A_{N}^{\alpha}$. Using (3) it is not difficult to show that $\exp _{x} \mid\left\{X \in M_{x}: X^{\alpha}=x^{\alpha}-A_{N}^{\alpha}\right\} \rightarrow$ (leaf through $z$ ) is a covering map. It follows that $\lim _{p \rightarrow \infty}\left(x^{\lambda}-X^{\lambda}(p)\right)$ exists. Thus $X_{1}^{i}=\lim _{p \rightarrow \infty} X^{i}(p)$ exists. Clearly, $z=$ $\exp _{x} X_{1}$, and so $\exp _{x}$ is onto $\bar{U}$ and hence the whole of $\bar{U}_{N}$. Moreover, it is easily seen from (3) that there is a neighborhood $W^{\prime}$ of $X$ such that $\exp _{x}: W^{\prime}$ $\rightarrow U_{N}$ is a diffeomorphism. A straightforward induction shows that $\exp _{x}: M_{x}$ $\rightarrow M$ is onto. The Walker atlas charts are evenly covered, and so $\exp _{x}$ is a covering map.

Case 2: $M$ is odd dimensional. There is a Walker atlas $\mathscr{A}$ on $M$ (see Walker [15]) such that in each chart the metric tensor has the form

$$
\left(g_{i j}\right)=\left[\begin{array}{ccc}
0 & 0 & I_{r} \\
0 & \pm 1 & 0 \\
I_{r} & 0 & C(t)
\end{array}\right] .
$$

Each chart has coordinates $(x, z, t) \in R^{r} \times R \times R^{r}$, and on the overlap of two charts the coordinates $\left(x_{*}, z_{*}, t_{*}\right)$ and $(x, z, t)$ are related by equations of the form

$$
x_{*}=x+\alpha_{1}(t) z+\alpha_{2}(t), \quad z_{*}= \pm z+\beta(t), \quad t_{*}=t+\gamma
$$


where $\gamma \in R^{r}$ is constant. The result now follows by an exactly analogous method to case 1 .

Corollary. If $M$ is a simply-connected complete Riemann m-manifold with a parallel framing of maximum nullity, then $M$ is diffeomorphic to $R^{m}$.

If we do not assume that $M$ is complete, we can still obtain strong results when $M$ is closed by appeal to the following theorem which strengthens a result of D. Tischler [14].

Theorem 8. Let $M$ be a smooth closed connected m-manifold which has a foliation $\mathscr{F}$ of codimension $r$, determined by $r$ independent closed, nonvanishing 1-forms $w^{1}, \cdots, w^{r}$. Then all the leaves of $\mathscr{F}$ are diffeomorphic, and there is a bundle map $f: M \rightarrow T^{r}$ such that if $F$ is the fibre and $L$ is a typical leaf, then $F \times R^{r}$ and $L \times R^{r}$ have the same universal cover and $\pi_{1}(F)$ is isomorphic to an extension of a subgroup of $\pi_{1}(L)$ by $Z^{n}$ (where $Z^{n}$ is the free abelian group on $n$ generators). Furthermore, if $L$ is simply connected, then $\pi_{1}(M)$ is abelian.

Proof. The 1 -forms $w^{\alpha}, \alpha=1, \cdots, r$, can be used to define a bundle-like metric $h$ for $\mathscr{F}$ (see [6]). It follows that there are $r$ independent orthonormal vector fields $X_{\alpha}$ spanning the normal distribution and satisfying $w^{\alpha}\left(X_{\beta}\right)=\delta_{\beta}^{\alpha}$.

Let $X=\xi^{\alpha} X_{\alpha}$ be a nonzero combination with $\xi^{\alpha}$ constant. Then the 1-parameter group of diffeomorphisms $\psi: R \times M \rightarrow M$ associated with $X$ corresponds to a geodesic flow normal to the leaves. But $h(X(x), X(x))$ is constant as $x$ varies over $M$, and so $\psi(s):, M \rightarrow M$ sends leaves to leaves for each $s \in R$. By varying the $\xi^{\alpha}$ it follows easily that all the leaves are diffeomorphic.

Denote the $r$-tuple of 1 forms $\left(w^{1}, \cdots, w^{r}\right)$ by $w$. Thus $\tau \mathscr{F}$ is defined by $\boldsymbol{w}=0$. Operations on $\boldsymbol{w}$ will be carried out component-wise. Let $a \in M$, and consider $H_{a}=\left\{[\sigma]:[\sigma] \in \pi_{1}(M, a), \int_{\sigma} \boldsymbol{w}=0\right\}$. Clearly $H_{a}$ is a normal subgroup of $\pi_{1}(M, a)$, and moreover it contains the commutator subgroup $C$. Following Rosenberg [11], let $\tilde{M}$ be the connected covering space of $M$ with respect to the group $H_{a}$. Then $\tilde{M}$ is a regular covering space of $M$ with covering group isomorphic to $\pi_{1}(M, a) / H_{a}$. Denote the covering projection by $p$. Defined on $\tilde{M}$ is the $r$-tuple $\boldsymbol{w}^{*}=p^{*} \boldsymbol{w}=\left(p^{*} w^{1}, \cdots, p^{*} w^{r}\right) . w^{*}$ is never zero and $d w^{*}=0$. Let $\mathscr{F}^{*}$ be the foliation determined by $\boldsymbol{w}^{*}$, and $\sigma$ a smooth closed curve in $\tilde{M}$ based at some point $\tilde{a}$ in $p^{-1}(a)$. Now, $\int_{\sigma} \boldsymbol{w}^{*}=\int_{p \cdot \sigma} \boldsymbol{w}$, and because $[p \cdot \sigma]$ represents an element in $H_{a}$ it follows that $\int_{\sigma} w^{*}=0$. Thus the integral of $\boldsymbol{w}^{*}$ about any closed curve in $\tilde{M}$ is zero, and so $\boldsymbol{w}^{*}=d \boldsymbol{g}$ where $\boldsymbol{g}$ is an $r$-tuple $\left(g^{1}, \cdots, g^{r}\right)$ of smooth real valued functions on $\tilde{M}$. The level surfaces of $g$ are precisely the leaves of $\mathscr{F}^{*}$.

The vector fields $X_{\alpha}$ lift to $X_{\alpha}^{*}$ on $\tilde{M}$ so that $p^{*} w^{\alpha}\left(X_{\beta}^{*}\right)=\delta_{\beta}^{\alpha}$. Thus $X_{\beta}^{*}\left(g^{\alpha}\right)=\delta_{\beta}^{\alpha}$, and so if $g^{\alpha}=c^{\alpha}, \alpha=1, \cdots, r$, is a leaf of $\mathscr{F}^{*}$, then the flow of $\xi X_{\beta}^{*}$ for a real number $\xi$ takes this leaf to the leaf $g^{1}=c^{1}, \cdots, g^{\beta}=\xi+c^{\beta}, \cdots, g^{r}=c^{r}$. 
It follows that if $\gamma \in R^{r}$, then $g=\gamma$ is a leaf of $\mathscr{F}^{*}$. The map $1: \tilde{M} \rightarrow R^{r}$ defined by $1(x)=g(x)$ is thus a bundle map, and so $\tilde{M}$ is diffeomorphic to $L_{0} \times R^{r}$ where $L_{0}$ is a leaf of $\mathscr{F}^{*}$. For each $\gamma \in R^{r}, L^{0} \times \gamma$ corresponds to a leaf of $\mathscr{F}^{*}$. It may be assumed without loss of generality that $p\left(L_{0}\right)=L$, the leaf of $\mathscr{F}$ through $a$.

Define a map $q: L \rightarrow L_{0}$ as follows. Let $b \in L$, and $\tau:[0,1] \rightarrow L$ be a path from $a$ to $b$. Lift $\tau$ to a path $\tilde{\tau}$ in $L_{0}$ with initial point $\tilde{a}$. Put $q(b)=\tilde{\tau}(1)$. This map does not depend on $\tau$ because closed paths in $L$ are represented in $H_{a}$ and so lift to closed paths in $L_{0}$. Thus $p: L_{0} \rightarrow L$ is a diffeomorphism, and $\tilde{M}$ can be identified with $L \times R^{r} . p: L \times R^{r} \rightarrow M$ is a regular covering with covering group $G$, isomorphic to $\pi_{1}(M, a) / H_{a}$. Now because $p_{\sharp}$ is a monomorphism, it follows that $H_{a}$ may be identified with $\pi_{1}(L, a) \subset \pi_{1}(M, a)$. Thus $G \cong \pi_{1}(M, a) / \pi_{1}(L, a)$ and is abelian because $C \subset H_{a}$. To show that $G$ is free abelian a further lemma is required.

An oriented closed transversal to $\mathscr{F}$ is a smooth path $j: S^{1} \rightarrow M$ such that $w^{\alpha}\left(j_{*}(\partial / \partial t)\right), \alpha=1, \cdots, r$, are not all zero and all have constant sign for $t \in S^{1}$ $(=\{t \in R: t \sim t+1\})$.

Lemma. Under the hypotheses of the theorem an element of $\pi_{1}(M, a)$ can be represented by an oriented closed transversal if and only if it belongs to $\pi_{1}(M, a)-\pi_{1}(L, a)$.

This result is a generalization of a theorem of Moussu [3] for the case $r=1$, and his proof can be modified directly.

Corollary. $\pi_{1}(M, a) / \pi_{1}(L, a)$ is free abelian.

Proof. It is abelian because $C \subset H_{a}$.

To show it has no torsion let $[\sigma] \in \pi_{1}(M, a)$ and let $\overline{[\sigma]} \neq 0$ be its coset in $\pi_{1}(M, a) / \pi_{1}(L, a)$. By the lemma $[\sigma]$ is representable by an oriented closed transversal $\tau$ say. But $\tau^{k}$ is always an oriented closed transversal and thus $\left[\tau^{k}\right]=$ $[\sigma]^{k} \notin \pi_{1}(L, a)$, i.e., $\overline{[\sigma]^{k}} \neq 0$. q.e.d.

Hence $G$ is free abelian, and moreover is finitely generated because $M$ is compact.

Now by Theorem 1 of Tischler [14] the 1 -forms $w^{1}, \cdots, w^{r}$ determine a bundle map $f: M \rightarrow T^{r}$. If $F$ is the fibre, then $F$ is closed and there is no loss of generality in assuming that $F$ is connected, because otherwise one could construct a $k$-fold cover ( $k=$ number of components of $F$ ) of $T^{r}$ (which is diffeomorphic to $T^{r}$ ) and a new bundle map onto this covering torus, with connected fibre.

Let $M^{\prime}$ be a simply connected cover of $M$. Then there is a regular covering $\phi: M^{\prime} \rightarrow L \times R^{r}$ such that $p_{0} \phi: M^{\prime} \rightarrow M$ is the projection. Let $F(a)$ be the fibre of $f$ through $a$, and $F_{1}$ the component of $p^{-1}(F(a))$ through $\tilde{a}$. Then $p: F_{1} \rightarrow$ $F(a)$ is a regular covering with covering group $G^{\prime}$ a subgroup of $G$. Clearly $G^{\prime}$ is finitely generated free abelian, i.e., $G^{\prime} \cong Z^{n}$ for some $n$. Since $\pi_{1}\left(L \times R^{r}\right)$ $=\pi_{1}(L)$, it follows that the covering group of $\phi$ is isomorphic to $\pi_{1}(L)$.

Let $F_{2}$ be a connected component of $\phi^{-1}\left(F_{1}\right)$. Then $\phi: F_{2} \rightarrow F_{1}$ is a regular 
covering with covering group isomorphic to a subgroup $A$ of $\pi_{1}(L)$. If $F_{2}$ is simply connected, then $\pi_{1}(F)$ will be isomorphic to an extension of $A$ by $G^{\prime}$ and the theorem will follow.

Let $\xi: R^{r} \rightarrow T^{r}$ be the regular covering induced by the standard $Z^{r}$ action. Then $\xi$ gives a pull back bundle of $M$ over $R^{r}$ with fibre $F$. But since $R^{r}$ is contractible, this bundle is trivial and so there is a covering map $\eta: F \times R^{r} \rightarrow$ $M$ such that for each $t \in R^{r}, \eta \mid F \times t$ is a diffeomorphism onto a fibre of $f$ in $M$.

Let $F^{\prime}$ be a simply connected cover of $F$. Then there is a covering map $\phi^{\prime}: F^{\prime} \times R^{r} \rightarrow F \times R^{r}$. Clearly $\eta \cdot \phi^{\prime}: F^{\prime} \times R^{r} \rightarrow M$ is a simply connected cover. Hence by uniqueness there is a homeomorphism $\lambda: F^{\prime} \times R^{r} \rightarrow M^{\prime}$ such that $(P \cdot \phi) \cdot \lambda=\eta \cdot \phi^{\prime}$. Thus $\lambda: F^{\prime} \rightarrow F_{2}$ is a homeomorphism and so $F_{2}$ is simply connected. This proves the first part of the theorem.

The second part follows immediately from the fact that $C \subset \pi_{1}(L, a)$ and so $C$ is trivial if $L$ is simply connected.

The next result is a direct application of this theorem.

Theorem 9. Let $M$ be a closed connected Riemann $2 r$-manifold with a parallel framing of type $(r, 0)$. Then each of the following statements is true:

(i) $M$ is covered by $R^{2 r}$.

(ii) The leaves of $\mathscr{F}$ are all diffeomorphic to $T^{q} \times R^{r-q}$ for some fixed $q \leq r$.

(iii) There is a bundle map $f: M \rightarrow T^{r}$ with fibre $F$ a closed connected $r$ manifold for which $F \times R^{r}$ is covered by $R^{2 r} . \pi_{1}(F)$ is isomorphic to an extension of $Z^{k}$ by $Z^{h}$ for some $k$ and $h$ with $k \leq q$.

(iv) $\pi_{1}(M)$ is isomorphic to an extension of $Z^{q}$ by $Z^{s}$ for some $s$.

(v) If $q=r$, then $F$ is diffeomorphic to $T^{r}$; and if $q=0$, then $M$ has the homotopy type of $T^{m}$, and is homeomorphic to $T^{m}$ for $m \neq 4$.

Proof. (ii)(iii)(iv) follow directly from previous theorems.

(i) follows from the fact that $M$ is covered by $T^{q} \times R^{r-q} \times R^{r}$.

(v) If $q=r$, then the leaves are closed, and since the foliation has trivial holonomy and bundle like metric it follows that $\mathscr{F}$ admits a bundle structure. Thus one may assume that $F$ is diffeomorphic to $T^{r}$. If $q=0$, then $\pi_{1}(M)$ is abelian. Since $M$ is compact and covered by $R^{2 r}$, it follows that $\pi_{1}(M)$ is finitely generated free abelian, i.e., $\pi_{1}(M) \cong Z^{k}$ for some $k$. Hence $M$ has the homotopy of $T^{k}$. Homology considerations and the compactness of $M$ show that $k=$ $m$. Hence by results of C. T. C. Wall [19] $M$ is homeomorphic to $T^{m}$ if $m \neq 4$.

Corollary. Let $M$ be a closed connected Riemann 4-manifold with a parallel framing of type $(2,0)$. Then $M$ is a $T^{2}$ bundle over $T^{2}$.

Proof. There are three cases, $q=0,1,2$. Since $F \times R^{2}$ is covered by $R^{4}$, it follows that $F$ is covered by $R^{2}$. If $q=0$, then $\pi_{1}(F)$ is free abelian, and so $F$ is diffeomorphic to $T^{2}$. If $q=2$, the result follows from the theorem.

If $q=1$, then $\pi_{1}(F)$ is at worst isomorphic to an extension of $Z$ by $Z^{h}$ for some $h$. Thus there is a regular $Z^{h}$ cover $p: F^{\prime} \rightarrow F$ where $\pi\left(F^{\prime}\right) \cong Z$. But $F$ 
is orientable (because $H_{1}(F ; Z)$ is torsion free), and so $F^{\prime}$ is orientable. It can be shown that $F^{\prime}$ is homeomorphic to $S^{1} \times R$. Identify $F^{\prime}$ with $S^{1} \times R$.

Let $x \in S^{1} \times 0 \subset F^{\prime}$, and $\sigma: S^{1} \times 0 \rightarrow F^{\prime}$ be the inclusion. Then $[\sigma]$ represents a generator of $\pi_{1}(\tilde{F}, x)$. Let $\psi$ be any orientation-preserving homeomorphism of $S^{1} \times R$. Then $\psi \cdot \sigma$ is an embedded $S^{1}$, and hence if $y=\psi(x)$ and $\tau:[0,1]$ $\rightarrow F^{\prime}$ is a path joining $x$ to $y$, then $\left[\tau^{-1} \cdot(\psi \cdot \sigma), \tau\right]=[\sigma]$. Thus $\sigma^{-1} \cdot \tau^{-1} \cdot(\psi \cdot \sigma) \cdot \tau$ is null homotopic. It follows easily that the commutator subgroup of $\pi_{1}(F)$ is trivial, and hence $F$ is diffeomorphic to $T^{2}$.

We now construct an example of a nontrivial $T^{2}$ bundle over $T^{2}$, which admits a parallel framing of type $(2,0)$.

Take $R^{4}$ with coordinates $(x, y, z, t)$ and metric $d s^{2}=2 d \times d z+2 d y d t$. With respect to this metric the fields $\partial / \partial x$ and $\partial / \partial y$ are mutually orthogonal, parallel and null. Consider the group $G$ of transformations of $R^{4}$ generated by $A, B, C, \theta$ defined as follows:

$$
\begin{aligned}
& A(x, y, z, t)=(x+1, y, z, t), \\
& B(x, y, z, t)=(x, y+1, z, t), \\
& C(x, y, z, t)=(x, y, z, t+1) \\
& \theta(x, y, z, t)=(x+t, y-z, z+1, t) .
\end{aligned}
$$

It is not difficult to show that $G$ is a properly discontinuous group of transformations of $R^{4}$ leaving the metric invariant. Since $\theta$ does not commute with $C, G$ is nonabelian.

Let $M=R^{4} / G$. Then $M$ admits a pair of mutually orthogonal, null parallel vector fields with respect to the induced metric, which lift to $\partial / \partial x$ and $\partial / \partial y$ on $R^{4}$. Furthermore, $M$ is compact, and the fields $\partial / \partial x, \partial / \partial y, z \partial / \partial x+\partial / \partial t$, $\partial / \partial z-z \partial / \partial y$ on $R^{4}$ are invariant under $G$ showing that $M$ is parallelizable.

The projection $\pi: R^{4} \rightarrow R^{2}$ defined by $(x, y, z, t) \rightarrow(z, t)$ is equivariant with respect to the action of $G$ on $R^{4}$ and the usual action of $Z^{2}$ on $R^{2}$, and so $\pi: R^{4} / G=M \rightarrow R^{2} / Z^{2}=T^{2}$ is well defined.

It is not difficult to show that $\pi$ gives a fibre bundle projection with fibre $T^{2}$ and structure group a subgroup of $T^{2} . M$ is not the trivial bundle because $\pi_{1}(M) \cong G \neq Z^{4}$.

\section{References}

[1] P. M. D. Furness \& T. J. Willmore, Parallel fields of lines, Differential Geomery, in Honor of K. Yano, Kinokuniya, Tokyo, 1972, 111-119.

[2] S. Kobayashi \& K. Nomizu, Foundations of differential geometry, Vol. I, Interscience, New York, 1963.

[ 3 ] R. Moussu, Feuilletage sans holonomie d'une variété fermée, C. R. Acad. Sci. Paris 270 (1970) 1308-1311.

[4] E. M. Patterson, Simply harmonic Riemann extensions, J. London Math. Soc. 27 (1952) 102-107. 
[ 5 ] E. M. Patterson \& A. G. Walker, Riemann extensions, Quart. J. Math. Oxford Ser. 3 (1952) 19-28.

[6] B. L. Reinhart, Foliated manifolds with bundle-like metrics, Ann. of Math. 69 (1959) 119-132.

[7] G. de Rham, Sur la reductibilité d'un espace de Riemann, Comment. Math. Helv. 26 (1952) 328-344.

[ 8 ] S. A. Robertson, Grid manifolds, J. Differential Geometry 4 (1970) 245-253.

[ 9 ] $\longrightarrow$, Twisted local-product manifolds, Proc. Edinburgh Math. Soc. (2) 17 (1970) 59-63.

[10] 161-167.

[11] H. Rosenberg, Actions of $R^{n}$ on manifolds, Comment. Math. Helv. 41 (1966) 170 178.

[12] H. S. Ruse, On parallel fields of planes in a Riemannian space, Quart. J. Math. Oxford Ser. 20 (1949) 218-234.

[13] - Parallel planes in a Riemannian $V_{n}$, Proc. Roy. Soc. Edinburgh, Sect. A, 58 (1950) 78-92.

[14] D. Tischler, On fibering certain foliated manifolds over $S^{1}$, Topology 9 (1970) 153-154.

[15] A. G. Walker, Canonical form for a Riemannian space with a parallel field of null planes, Quart. J. Math. Oxford Ser. 1 (1950) 69-79.

[16] Canonical forms (II) : parallel partially null planes, Quart. J. Math. Oxford Ser. 1 (1950) 147-152.

[17] - Riemann extensions of non-Riemannian spaces, Convegno Internaz. Geometria Differenziale (Italia, 1953) Cremonese, Rome, 1954, 64-70.

[18] H. Wu, On the de Rham decomposition theorem, Illinois J. Math. 8 (1964) 291311.

[19] C. T. C. Wall, Surgery on compact manifolds, Academic Press, New York, 1970.

[20] A. Weinstein, Symplectic manifolds and their Lagrangian submanifolds, Advances in Math. 6 (1971) 329-346. 